\title{
Critical Analysis of a Field Research Report on ADD and ADHD
}

\section{André Michaud*}

SRP Inc., Canada

\begin{abstract}
Analysis of a research report shedding a disquieting light on the reasons that were identified for diagnosing a constantly increasing number of children as suffering from ADD/ADHD and subsequent prescription of Ritalin and other psychostimulants.
\end{abstract}

Keywords: Psychostimulants; ADD; ADHD; Elementary school; Literacy

\section{Introduction}

The present analysis abstracts the main conclusions of an exhaustive field study carried out by a Canadian research team regarding out of control ADD and ADHD diagnosing and psychostimulants prescription to elementary school children in the city of Laval, Canada [1] which appears to be a reflection of the diagnosing and prescription trend in North America. Complete identification of the research report is given in Appendix A.

To the study authors' knowledge, this study was the first exhaustive attempt to estimate the extent of use of psychostimulant drugs in relation with $\mathrm{ADD} / \mathrm{ADHD}$ diagnosis for elementary school children to have been carried out in Canada. Its objectives were:

1. To describe the perceptions of the various actors involved in the follow up of the cases of children who have been diagnosed with ADD/ADHD,

2. To estimate the extent of psychstimulant drugs prescription to elementary school children in the city of Laval.

The study reports that the psychiatric label ADD/ADHD, which stands for Attention Deficit Disorder/ Attention Deficit and Hyperactivity Disorder, stems from another label, that of "minimal brain dysfunction" originating from an American study that was conducted in 1960's. This study attempted to identify, without success, whether learning problems observed in some children could be due to some brain pathology.

The Cohen et al.'s research report [1] provides an historical recap of the development of the concept of "Attention Deficit Disorder/ Attention Deficit and Hyperactivity Disorder", or ADD/ADHD, of its scientific foundations and of the prevailing opinions regarding its causes in medical circles.

The systematic non-involvement of two groups of scientists whose contribution could be critical is noted with regards to the development of this concept, they are the communities of neurophysiologists and doctors in pedagogy.

The study mentions that a preliminary study carried out in 1997 in the province of Québec, Canada [2] showed that a constantly increasing number of children were diagnosed as being "hyperactive" and subsequently medicated in the province's elementary schools.

It was observed that the school system submitted parents and physicians alike to enormous pressure to medicate these children.

The current analysis will bring to light the helplessness of the actors to take control of the situation, the actions of each actor being the outcome of the actions of the others, which apparently creates synergy causing the constant increase in psychostimulant prescription, despite their proven inability to improve the condition of children except for an observed calming effect, and that no data exists confirming any relation between ADD/ADHD and any brain dysfunction.

Despite a voiced intention by all actors to help these children, this effective means made available to calm "troublesome" children seems to have become socially accepted and freed of guilt, and appears to have become over time the last resort tool of choice to maintain order in classrooms in the school system, given that children so medicated become passive and that most of them are typically not provided with complementary help beyond the psycho stimulant prescription itself, mainly consisting of Ritalin (methylphenidate, $85-90 \%$ of cases), but also of Dexedrin (dextroamphetamin) and Cylert (pemolin).

It was also observed that prescription of psychostimulant drugs increases so quickly that statistics are already obsolete when published in scientific journals.

Health professionals are so overwhelmed by the sheer number of requests that the risk of misdiagnosis has become extreme for all children who attract attention in the school system since the official diagnosis criteria for $\mathrm{ADD} / \mathrm{ADHD}$ are strictly behavioural.

The Cohen et al.'s report indicates that the procedure does not even involve that the child be examined by a physician for the diagnosis to be established, which means that it is established mainly on the basis of information obtained from teachers and parents to the effect that the behavioural traits associated "by them" to $\mathrm{ADD} / \mathrm{ADHD}$ are manifested by the child.

This means that children who "disturb the peace" by being too enthusiast, or attract attention by becoming restless due to feeling bored in class, are at high risk of being misdiagnosed ADD/ADHD, without being examined by competent professionals. The study concludes that once the evaluation process has been triggered, the majority of children manifesting a range of behavioural traits of various origins WILL eventually be medicated, and it is unfortunately observed that

*Corresponding author: André Michaud, Individual Senior Researcher, SRP Inc. Canada, Tel: 14186240608; E-mail: srp2@srpinc.org

Received December 06, 2016; Accepted December 08, 2016; Published December 15, 2016

Citation: Michaud A (2016) Critical Analysis of a Field Research Report on ADD and ADHD. Int J Swarm Intel Evol Comput 5: 142. doi: 10.4172/2090-4908.1000142

Copyright: @ 2016 Michaud A. This is an open-access article distributed under the terms of the Creative Commons Attribution License, which permits unrestricted use, distribution, and reproduction in any medium, provided the original author and source are credited. 
psychostimulant medication is immediately considered as soon as a child is first perceived as presenting a problem in class, whatever the cause.

\section{ADD/ADHD, the Origins of the Concept and its Foundations}

Even if the 1960 American study failed to identify any brain dysfunction to explain ADD/ADHD associated behaviour, numerous behavioural traits were identified and associated with these labels, while various drugs were experimented with to potentially remedy the condition of diagnosed children.

Thirty years later, a final text of the Consensus Conference on ADD/ADHD held in November 16th to 18th of 1998 at the United States National Institute of Health was published [3] that declared unequivocally that: "There are no data to indicate that $A D D / A D H D$ is due to a brain malfunction". The report concludes moreover, that no objective testing procedure allows singling out any child as being affected by this problem, and that the official diagnosis criterion of $\mathrm{ADD} / \mathrm{ADHD}$ is strictly behavioural.

Despite this final text's conclusions, in excess of 5 million American children in 1997 (9 million in 1999) and tens of thousands of FrenchCanadian children are needlessly diagnosed ADD/ADHD and prescribed psychostimulants to control behavioural traits that have no recognized medical foundation.

\section{The Effects of Psychostimulants}

An important study published in 1993 [4], summarizes the results of the thousands of other studies carried out on the effects of psychostimulants on children: This summarizing study shows that all children diagnosed or not, as well all animals on which the drugs were tested, display the same reaction the drugs. It was found that psychostimulants increase concentration and effort while minimizing impulsivity and increasing obedience for a short initial period going from 7 to 18 weeks in all subjects tested, a period after which all effectiveness was lost.

It was found that the medication did not improve complex abilities such as reading, nor social interaction. The medicated children's results in school showed improvement, but children's observed various learning disabilities were not corrected by the medication.

There was no improvement noted for any significant emotional problem. No evidence was found of any long term improvement in academic results or any decrease of antisocial behaviour for children submitted to psychstimulant prescription.

The known negative side effects of psychstimulant medication are a decrease in appetite, sleeplessness, damaging effects on self-image and on cognition, such as lessening of creativity and spontaneity; appearance of stereotyped behaviour, nervousness, emotional instability, restlessness, head and belly aches, thwarting of the growth curve, and on occasions, increase or emergence of verbal or motor mannerisms or motor twitches, that in some cases reached the level of psychotic behaviour.

\section{The Pressure from the School System to Diagnose and Medicate}

Here are the main points determining the diagnosis and prescription of the medication: From the Cohen et al.'s analysis, it was concluded that the various problems that teachers identify as possible symptoms of $\mathrm{ADD} / \mathrm{ADHD}$ during the first year of elementary schooling typically are disturbances in the classroom, failure, inattention and a variety of behavioural problems.

Teachers then refer children drawing such attention for psychological evaluation, and inform parents that their child should be evaluated by a physician. The study shows that teachers are the initiators of the process, but that they are only minimally involved afterwards.

Parents tend to resist considering medication and the child is eventually referred to a specialist for medical evaluation (paediatrician or neurologist) after the child has been evaluated by the psychosocial authority in the school.

The report concludes that physicians are generally constrained by circumstances to diagnose children on the basis of psychosocial or psychological evaluations that they deem incomplete or unsatisfactory. Physicians report that they tend to be overloaded with cases and that they cannot avoid being sensitive to the pressure that the school exerts on parents.

The schools' psychosocial personnel are critical of the rigor of the medical evaluation while front line physicians experience difficulties in establishing a proper diagnosis, considering the absence of biological criteria, which adds to the contradictions between the various evaluations done by the parents, the teachers and the school psychosocial personnel. Specialists on their part harshly criticize the lack of knowledge of the psychosocial school personnel.

All of the actors are aware of a lack of communication between the various groups, and note that resources are minimal after the diagnosis. A lack of support is observed for all actors and the families. But most critically, there is a lack of follow up for the medicated children.

"Family physicians and specialists alike are unanimous regarding the pressures exerted by the school on parents for them to consult a physician. Consequently, parents understand that they must consult a physician, or else their child will be removed from the class and suspended from the school" [1]. The pressure that the school exerts on parents to prescribe psychostimulants is so acute in many cases that some even ask physicians to "reassure" the school teachers and psychosocial personnel regarding the intellectual abilities of their children.

The acuteness of the pressures exerted by the school on parents is a major problem for physicians, coupled with the pressure that the school directly exerts on them. Physicians who tend not to give a positive diagnosis and do not prescribe the medication tend to end up not being referred children, because school authorities then end up referencing the names of physicians that are more open to diagnose positively and medicate [1].

Frontline physicians and specialist assert that once medicated, the school withdraws all support to the child and his family. One of the physicians who were party to the study went as far as stating that " $a$ school problem has been medicalized because the school has abandoned its primordial role towards children."

It is observed that the medication should not be the only action taken, but that it factually is. It is observed that medication is used as a pretext to diminish services to children, because none of the actors feels capable of taking on the responsibility of following up medicated children, in the current situation.

The availability of the medication and the insistence of the school that it be used even without complementary resources force the actors to act against their convictions. The interviews of the various actors bring to light that each actor feels helpless, isolated and unable to 
escape the pressure to prescribe psychostimulants that emanates from the other actors or the school.

The final conclusion is so damning that the authors have expressed it in the form of a question: "Is medication used mainly to restore "order" and "calm" in the system?"

\section{Conclusion}

It can also be wondered if the extremely restrictive budgetary policies applied for years at all levels of the schooling system by the Quebec government could be a driving factor in this explosion in the number of "declarations" of cases of hyperactivity, which coincided with the establishment of these policies (which is a point not covered by the study), because these policies are such as to make the amounts allocated to schools by the Ministry to support each child "declared" in difficulty is superior to the amount allocated for children who are not declared in difficulty, since this policy applies even when the diagnosed children receive no extra service besides the actual psychostimulant prescription, for which the school incurs no costs at all.

It was established in the 1950's by French neurophysiologist Chauchard [5] that an inadequate activation of the brain verbal centers between birth and the age of approximately 7 , systematically results in an incomplete development of children's verbal centers, which can cause a significant thwarting in the development of the ability of verbal comprehension and expression:

"Once the normal age for the development of the verbal areas is passed, such training will become difficult. The fundamental law of cerebral development, that is, the possibility of possessing later a completely normal brain, endowed with all of the human aptitudes, requires that cerebral maturation always takes place in an environment, not only physical, but cultural and affective, that will favor it. Nothing can be done too soon, but soon, it is too late" [5].

The consequence of belated development of all verbal areas of the brain can then obviously only be an increased level of difficulty for these children to clearly understand teachers' explanations after they reach school age, inducing a state of inattention, which can be an obvious cause of learning problems in school and of the disturbing behavioral traits that are now associated with ADD/ADHD.

The question now comes to mind as to what repercussions the Cohen et al.'s 1999 study [6] had in the local educational community. In 2015 , that is, 16 years after the report was published, it seems that prescription of Ritalin and other psychostimulants has reached unheard of records in the province of Quebec [7], which troublingly parallels the constantly increasing illiteracy curve in this Canadian province, which in turn elicits the following question: Can psychostimulant prescription increase in the absence of sufficient early verbal stimulation be linked to the increase in illeteracy in the province of Quebec?

The OECD figures are unforgiving with regard to illiteracy in this province, revealing that in 1994, the functional illiteracy rate was $38 \%$ in the Quebec province, had risen to $50 \%$ by 2005 and reached an incredible rate of 53\% in 2013 [8], which suggests, since both issues are linked to children's learning problems, that the prescription of psychostimulants, with or without complementary help, besides their known tendency to induce unfortunate negative side effects in medicated children, does not improve the condition of children as confirmed by the 1993 study [4] except for an observed calming effect, which leads to conclude, according to the Cohen et al. report, that the only benefit of such psychostimulant prescription is that order is better maintained in classrooms when disturbing children are medicated.
It is consequently quite unfortunate that in 2016, 50 years after the sound practice of teaching children to read to proficiency during the first year of primary school was abandoned in the province of Quebec, as new educational theories were implemented as part of a major educational reform [9], that do not require early mastering the reading skill, close to 100 local experts reunited in congress in Quebec city December 1 and 2 of 2016 [10], unanimously agreed on the need to intervene early in the life of children to detect their "learning problems".

But no mention was reported to the effect that such "learning problems" concerned "insufficient mastery of all aspects of language", nor that re-establishment of systematic early teaching of the reading skill was considered, which can hardly lead, when correlated with Chauchard's findings, to anything but more ADD/ADHD diagnoses and phychostimulant prescription.

On the other hand, it is constantly observed that when children are properly taught to read to proficiency before the age of 7 [6], children remain calm in relation with the norm of their age, in accordance with Chauchard's conclusions, because they progressively think and analyze more and more clearly as they progressively master with more refinement the language that they think with and thus become progressively better equipped to understand and control the progressively increasing complexity of the challenges that they are confronted with as they grow up [11].

\section{Appendix A}

The field research report is in French and its title translated to English is: "Attention Deficit/Hyperactivity: Perception of the Authors and Use of Psychostimulants". The original title is: "Déficit d'attention/ hyperactivité: Perception des auteurs et Utilisation de psychostimulants" [1]. The research team was comprised of: David Cohen, Ph.D., Irma Clapperton, M.D., M.Sc., CSPQ, Pauline Gref, M.D., FRCPC and Yves Tremblay, M.D., CSPQ, FRCPC. And the project also involved the collaboration of Sylvie Cameron, M.A.

\section{References}

1. Cohen D, Clapperton I, Gref P, Tremblay Y (1999) Attention deficit/hyperactivity: Perception of actors and use of psychostimulants. RRSSS Laval, Canada.

2. Doré C, Cohen D (1997) The prescription of stimulants to "hyperactive" children. Mental Health in Quebec 22: 216-328.

3. Diagnosis and treatment of attention deficit hyperactivity disorder (1998) National institutes of health consensus statement.

4. Swanson JM, McBummet K, Wigal T, Pfiffner LJ, Lerner MA (1993) Effect of stimulant medication on children with attention deficit disorder: A review of reviews. Exceptional Children 60: 154-162.

5. Chauchard $P(1960)$ The brain and consciousness. Editions du Seuil, France.

6. Michaud A (2003) The neurolinguistic foundation of intelligence. SRP Books.

7. Philippe M (2015) Ritalin: Consumption reaches record highs in Quebec.

8. (2015) Québec report of the program for the international assessment of adult competencies (PIAAC). Quebec Institute of Statistics.

9. Michaud A (1999) Our Bankrupt Elite. SRP Books. Smashwords Revised in 2012.

10. Patricia C (2016) 5 Ideas for changing the world in education. Press Releases.

11. Michaud A (2016) Comprehension process overview. J Biom Biostat 7: 317. 